\title{
Trauma de tórax. Experiencia de cinco años en el Centenario Hospital Miguel Hidalgo
}

\author{
Flores-Álvarez E*, García-Ortega DY**, Jiménez-Fernández C*, Luna-Sánchez A**
}

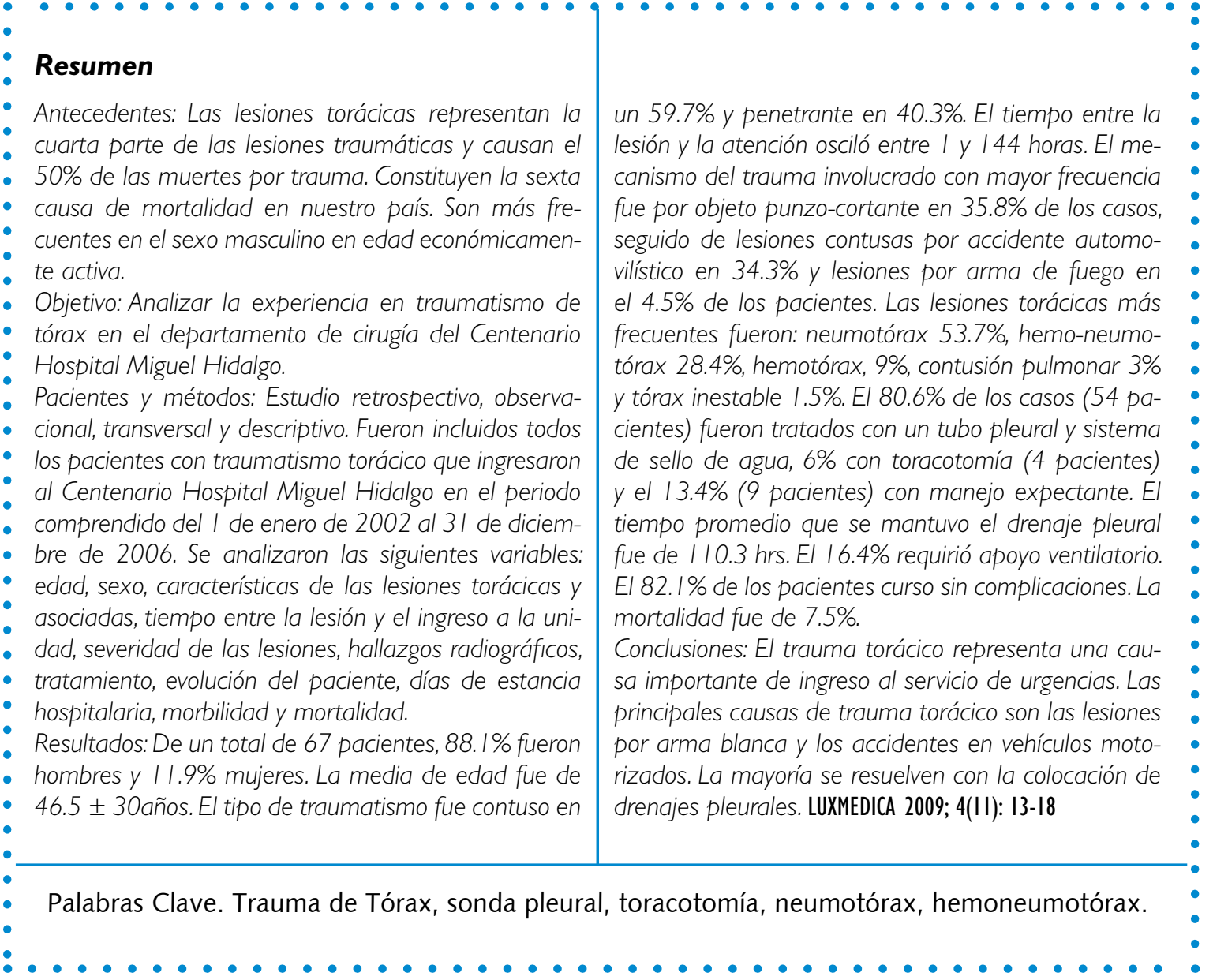

* Maestro en Ciencias, Cirujano General, Cirujano Oncólogo, Profesor Titular del Posgrado en Cirugía General.

** Médico Residente de Cirugía General. Departamento de Enseñanza e Investigación. Centenario Hospital Miguel Hidalgo, Aguascalientes, Ags. 


\section{Introducción}

Las lesiones traumáticas constituyen hoy en día la sexta causa de mortalidad en nuestro país, únicamente por debajo de las enfermedades crónico-degenerativas y neoplásicas. Representan la causa más frecuente de mortalidad en personas económicamente activas, siendo más frecuentes en el sexo masculino ${ }^{1}$.

Las lesiones del tórax corresponden a una cuarta parte de las lesiones traumáticas y el $50 \%$ de las muertes por trauma. Las lesiones torácicas se clasifican en: trauma cerrado, penetrante o ambos; que generalmente ocurren como resultado de accidentes automovilísticos, lesiones por proyectil de arma de fuego o punzo-cortante, caídas de altura o compresión torácica por aplastamiento. La incidencia de lesiones del tórax por trauma penetrante es aproximadamente del $96 \%$, mientras que la de las lesiones cerradas es del $4 \% .2,3,4$ Las estructuras torácicas lesionadas pueden incluir: la pared torácica, los pulmones, el corazón, los grandes vasos y el diafragma. El neumotórax a tensión, el hemotórax masivo y el taponamiento cardíaco son lesiones potencialmente mortales que pueden ocurrir como resultado de un trauma de tórax. El tratamiento de estos pacientes se hace bajo los lineamientos establecidos por el ATLS (Advanced Trauma Life Support). ${ }^{5}$

El diagnóstico y tratamiento de los traumatismos torácicos depende en su mayor parte de la magnitud de la lesión y de la evidencia de lesiones asociadas. En la mayoría de los pacientes con lesiones por trauma de tórax resulta factible su resolución en forma conservadora, manejados con buenos resultados mediante el drenaje de cavidad pleural con tubos pleurales. ${ }^{6,7,8}$ En el trauma se presentan tres picos de mortalidad: uno inicial, que se da en los primeros minutos al trauma, el cual aporta un $50 \%$ de la mortalidad global; un segundo pico ocurre en las 3 horas siguientes al trauma, ocurren $20 \%$ de las muertes, y un tercer pico que corresponde a la mortalidad tardía y representa el 30\% de la mortalidad. ${ }^{10,11}$ Se ha considerado a la primera hora luego del trauma como el "periodo de oro", durante el cual, con un manejo adecuado se puede reducir la mortalidad en el segundo y tercer pico de la mortalidad por trauma.

Las caídas y los accidentes vehiculares son las causas más comunes de traumatismo torácico en la literatura internacional. ${ }^{6-8,12}$ En nuestro medio aparecen como una de las causas mas frecuentes, aunadas a las lesiones producidas por arma blanca, producto de riñas y asaltos.

El objetivo del trabajo es analizar la experiencia en el manejo traumatismo de tórax en el departamento de cirugía de nuestro hospital en un periodo de 5 años. 


\section{Material y método}

Se realizó un estudio retrospectivo, observacional, transversal, descriptivo y analítico. Fueron incluidos todos los pacientes mayores de 16 años con diagnóstico de traumatismo torácico que ingresaron al Centenario Hospital Miguel Hidalgo del ISEA en el periodo comprendido del 1 de enero 2002 al 31 de diciembre de 2006. Las variables estudiadas fueron la edad, el género, las características de las lesiones torácicas, los hallazgos radiográficos, el tiempo entre la lesión y el ingreso a la unidad, el mecanismo del trauma, el tipo de lesiones torácicas, el grado de severidad de las lesiones, la presencia de lesiones ex- tratorácicas, el tratamiento efectuado en urgencias, el tratamiento en la Unidad de Terapia Intensiva, el tipo de tratamiento quirúrgico, el tiempo de permanencia del drenaje pleural, el desarrollo de complicaciones, los días de estancia hospitalaria y la mortalidad.

Se efectuó un análisis descriptivo de cada una de las variables en el paquete estadístico SPSS versión 17.0. Para las variables cualitativas nominales $u$ ordinales se calculó la frecuencia absoluta y porcentajes. Para las variables cuantitativas se estimaron medidas de tendencia central y de dispersión según el tipo de distribución de los datos.

|| || || || || ||||||||||||||||||||||||||||||||||||||||||||||||||||||||||||||||||||||||||||||||||||||||||||||||||||||||||||||||

\section{Resultados}

Fueron incluidos un total de 67 pacientes, el $88.1 \%(n=59)$ fueron hombres y el $11.9 \%$ $(n=8)$ mujeres. La media de la edad fue de $46.5 \pm 30$ años. El tipo de traumatismo fue contuso en 40 pacientes $(59.7 \%)$ y penetrante en 27 (40.3\%). El tiempo transcurrido entre el momento en que ocurrió la lesión y el de la atención medica osciló entre 1 a 144 horas, la mayoría acudió al hospital durante las primeras 3 horas (primera hora, $29.9 \%$; segunda hora, $26.9 \%$, $y$; tercera hora, 19.4\%). El mecanismo del trauma involucrado con mayor frecuencia fue por objeto punzo-cortante en 24 pacientes $(35.8 \%)$, accidente automovilístico en 23 pacientes (34.3\%) y por arma de fuego en 3 casos (4.5\%). Las causas según el tipo de traumatismo se muestran en la Tabla 1.

\section{Tabla I}

\section{Tipo de Trauma Torácico}

\begin{tabular}{|lcc|}
\hline \multicolumn{1}{c}{ Tipo } & Número de Casos & Frecuencia (\%) \\
Penetrante por objeto punzo-cortante & 24 & 35.8 \\
\hline Contuso automovilístico & 23 & 34.3 \\
\hline Contuso laboral & 6 & 9 \\
\hline Contuso por violencia física & 5 & 7.5 \\
\hline Otras contusiones & 6 & 9 \\
\hline Penetrante por proyectil de arma de fuego & 3 & 4.5 \\
\hline Total & 67 & 100 \\
\hline
\end{tabular}


Las lesiones torácicas más frecuentes fueron: neumotórax, 53.7\% $(n=36)$; hemo-neumotórax, $28.4 \% \quad(n=19)$; hemotórax, $9 \%(n=6)$; contusión pulmonar, $3 \%(n=2), y$; tórax inestable, $1.5 \%(n=1)$. El $34.3 \%(n=23)$ de los pacientes presentaron fracturas costales, el $4.5 \%$ contusión pulmonar $(n=3)$ y otro $4.5 \%(n=3)$ presentó más de dos lesiones. El 55.2\% $(n=37)$ de los pacientes no presentaron otras lesiones torácicas o extratorácicas asociadas.

Las lesiones extratorácicas asociadas fueron: fracturas óseas en 10 pacientes $(14,9 \%)$, traumatismo craneoencefálico en
6 pacientes $(9 \%$ ) y lesión a órganos abdominales en 3 pacientes (4.5\%). El $80.6 \%$ de los pacientes $(n=54)$ fueron tratados con sonda pleural y sistema de sello de agua, se realizo toracotomía solo en el $6 \%$ de ellos $(n=4)$ y se estableció un manejo conservador no invasivo en $13.4 \% \quad(n=9)$. En el $70.2 \%(n=40)$ de los casos el drenaje pleural fue mantenido por menos de 5 días, el $28.1 \%$ entre 5 y 10 días $(n=16)$ y en $1.7 \%(n=1)$ por más de 10 días.

Doce pacientes $(17.9 \%)$ ameritaron manejo en la Unidad de Cuidados Intensivos, el resto fueron manejados en piso con medidas generales.

\section{Tabla 2}

\section{Lesiones Torácicas y Extratorácicas}

\begin{tabular}{|lcc|}
\hline \multicolumn{1}{|c}{ Tipo } & Número de Casos & Frecuencia (\%) \\
Lesiones óseas & 10 & 14.9 \\
\hline Lesiones óseas + TCE & 6 & 9 \\
\hline Lesiones de órganos abdominales & 3 & 4.5 \\
\hline TCE & 1 & 4.5 \\
\hline Lesiones óseas + abdominales & 1 & 4.5 \\
\hline Lesiones torácicas + lesiones óseas & 1 & 4.5 \\
\hline Lesiones torácicas + abdominales & 1 & 4.5 \\
\hline Lesiones torácicas + abdominales + óseas & 1 & 4.5 \\
\hline
\end{tabular}

*TCE: Trauma cráneo encefálico

Solo 4 pacientes $(6 \%)$ desarrollaron complicaciones médicas a nivel torácico: síndrome de dificultad respiratoria del adulto (SDRA) en $3 \%(n=2)$, neumonía en $1.5 \%(n=1)$ y atelectasia en $1.5 \%(n=1)$.

Nueve $(13.4 \%)$ de los pacientes ameritaron uso de aminas vasoactivas y 11 pacientes $(16.4 \%)$ requirieron apoyo mecáni- co ventilatorio. La mortalidad fue de $7.5 \%$ $(n=5) ; 1$ defunción fue relacionada directamente con el trauma torácico y las otras 4 lesiones en otros órganos y/o complicaciones por co-morbilidades del paciente.

La gravedad del traumatismo torácico la evaluamos con la escala de severidad de la lesión (Injury Severity Scale).12,14-18 Fueron 
considerados como trauma moderado el $55.2 \%$ de los casos, trauma grave que no pone en riesgo la vida el $17.9 \%$, trauma grave que pone en riesgo la vida el $17.9 \%$ $y$, trauma crítico con supervivencia incierta el 9\%. Las complicaciones secundarias res- piratorias y hemodinámicas determinaron que el $17.9 \%$ de los pacientes requirieran ser manejados en la unidad de terapia intensiva, con una adecuada evolución en la mayoría de los casos.

| | | | | | | | | | | | | | | | | | | | | | | | | | | | | | | | | | | | | | | | | | | | | | | | | | | | | | | | | | | | | | | | | | | | | | | | | | | | | | | | | | | | | | | | | | | | | | | | | | | | | | | | | | | |

\section{Discusión}

Las lesiones traumáticas continúan siendo una causa frecuente de mortalidad e incapacidad en personas en edad productiva, lo cual constituye un costo importante en horas laborales así como pérdidas y gastos en atención médica. ${ }^{2}$ El trauma torácico representa una causa importante de consulta en los servicios de urgencias, la mayoría de los pacientes son del género masculino, debido a que los hombres se encuentran más expuestos a mecanismos violentos como riñas, agresiones físicas y accidentes automovilísticos. Las lesiones producidas por vehículo motorizado son, en la actualidad, la causa más frecuente de trauma torácico en la mayoría de los reportes de la literatura mundial; sin embargo, las lesiones por objetos punzo-cortantes son el mecanismo más frecuente por el que se producen las lesiones torácicas en los pacientes atendidos en nuestro hospital. Las lesiones por proyectil de arma de fuego continúan siendo una causa menos frecuente de trauma de tórax en nuestra institución, sin embargo, ésta ha ido aumentando en los últimos años.

El tratamiento basado en la inserción de tubo pleural y colocación de sello de agua, es la alternativa más utilizada en el caso de las lesiones traumáticas del tórax, en diversas series es reportada entre el 85 y $90 \%$ de los casos. ${ }^{3}, 4,9,13$ El tipo de lesión más frecuente en nuestros pacientes fue el neu- motórax; en su mayoría fueron adecuadamente tratados con la colocación de tubos pleurales y sistemas de sello de agua, que fueron retirados en los primeros 5 días.

La hemorragia intratorácica puede ser originada por lesiones de los vasos intercostales, mamarios o del parénquima pulmonar. La hemorragia secundaria a la lesión del parénquima pulmonar generalmente se autolimita debido a la baja presión en la circulación pulmonar, el alto contenido de tromboplastina del parénquima pulmonar y el efecto compresivo del pulmón colapsado sobre el sitio sangrante. ${ }^{2,4,5,13}$ En pocas ocasiones se requiere una toracotomía de urgencia para tratar el hemotórax; en nuestra serie sólo se realizó en el $6 \%$ de los pacientes.

El trauma torácico se puede acompañar de lesiones en múltiples sistemas, lo cual condiciona una alta mortalidad en este grupo de pacientes. En la presente serie encontramos que en el $35.8 \%$ de los casos presentaban lesiones extratorácicas. La asociación más importante fue la existente entre trauma torácico, lesiones óseas y traumatismo craneoencefálico, presente en el $9 \%$ de los pacientes. En general, los pacientes con trauma de tórax sin lesiones en otros órganos evolucionan sin complicaciones durante su internamiento $y$, aunque su estancia hospitalaria no es breve suelen egresarse antes del décimo día. 


\section{Condusiones}

El trauma torácico representa una causa importante de ingreso al servicio de urgencias en nuestro hospital. El trauma secundario a lesiones por arma blanca y vehículos motorizados representan las principales causas de trauma torácico en pacientes atendidos en nuestra institución. La mayoría de los casos se resuelven con la colocación de drenajes pleurales, sistemas de sello de agua y cuidados de soporte.

\section{Bibliografía}

1 Secretaría de Salud. Dirección General de Estadística e Informática. Mortalidad: México, SSA 2007.

2 Valenzuela DM, Cancino AP, Cabezas SF, Donoso DG, De la Torre I. Experiencia en traumatismo torácico. Hospital Valparaíso. Rev Chilena Cir 2003:55(5):4449-453.

3 Méndez-Acuña J. Incidencia de Cirugía Torácica Servicio de Cirugía Hospital Santa Bárbara. Rev Inst Med Sucre LXV 2000;117:54-57.

4 Guzmán F, Ramírez JC, Ramírez G, Rodríguez G, Supelano M, Cadena J, Escallon F, Holguín R. Trauma de Tórax. Rev Colombiana Cir. 1990;5(1):38-45

5 American College of Surgeons Commitee on Trauma: Advanced Trauma Life Support.

6 Karmy-Jones R, Nathens A, Jurkovich GJ, Shatz DV Brundage S, Wall MJ, Engelhardt S, Hoyt DB, Holcroft J, Knudson MM, Michaels A, Long W. Urgent and Emergent Thoracotomy for Penetrating Chest Trauma. J Trauma 2004;56:664-669.

7 Asensio JA, O'Shanahan G, Petrone P, Costa D, Robin-Lersundi A. Emergency thoracotomy: a critical evaluation of the technique. Cir Gen 2004;26:12837.

8 Cothren C, Moore EE. Emergency department thoracotomy for the critically injured patient: Objectives, indications, and outcomes. World J Emerg Surg. 2006:1:4

9 Morales-Wong MM, Gómez-Hernández MM, Ramos-Godines A, González-Folch R. Lesiones torácicas graves y el enfoque del control de daños. Rev Cub Cir 2008; 47(1)

10 www.fepafem.org.ve/Guias_de_Urgencias/Trauma/ Trauma_pulmonar.pdf
11 Llerena-Rodriguez M. Caracterización de la evaluación del traumatismo torácico. Rev Cub Med Int Emerg 2007;6(4):896-910.

12 Hans-Christoph $P$, Dierk R, Rice J, Ebisch $M$, Krettek C, Tscherne H. Appraisal of Early Evaluation of Blunt Chest Trauma: Development of a Standardized Scoring System for InitialClinical Decision Making. J Trauma 2000;49:496-504.

13 Lombardo-Vaillant TA. Manejo de los seis grandes del trauma torácico. Primera parte. Rev Cub Med Mil 2008;1:37.

14 Martinus R, Krettek C, Otte D, Wiese SM, Stalp M, Ernst S, Hans-Cristoph P. Correlation between Crash Severity, Injury Severity, and Clinical Course in Car Occupants with Thoracic Trauma: A Technical and Medical Study. J Trauma 2001; 50:10-16.

15 Copes WS, Champion HR, Sacco WJ, Lawnick MM, Keast SL, Bain LW. The Injury Severity Score Revisited. J Trauma 1988;28:69-77.

16 Champion HR, Copes WS, Sacco WJ, Lawnick MM, Bain LW, Gann DS, Gennarelli T, Mackenzie E, Schwaitzberg S. A New Characterization of Injury Severity. J Trauma 1990;30(5):539-546.

17 Baker SP, O'Neill B, Haddon W JR, Long WB. The Injury Severity Score: a method for describing patients with multiple injuries and evaluating emergency care. J Trauma 1974;14:187-96.

18 Agúndez-Meza JJ, Cardoza-Macías F. Comparación de ISS (Escala de severidad de lesiones) con resultado final del tratamiento. Cir Gen 2000;22(1):9-17. 